\title{
Outcome of Radiation Therapy for Stage IVB Uterine Cervical Cancer With Distant Lymph Nodes Metastases; Sequential Irradiation for Distant Lymph Nodes Metastases
}

\author{
YUKI MUKAI ${ }^{1}$, NAHO RUIZ YOKOTA ${ }^{2}$, MADOKA SUGIURA ${ }^{1}$, TAICHI MIZUSHIMA ${ }^{2}$, RISA TANIUCHI ${ }^{1}$, YUICHI \\ IMAI $^{2}$, KOTARO HASHIMOTO $^{1}$, YUYA TABUCHI ${ }^{1}$, ETUSKO MIYAGI $^{2}$ and MASAHARU HATA ${ }^{1}$ \\ ${ }^{1}$ Department of Radiation Oncology, Yokohama City University Graduate School of Medicine, Yokohama, Japan; \\ ${ }^{2}$ Department of Obstetrics and Gynecology, \\ Yokohama City University Graduate School of Medicine, Yokohama, Japan
}

\begin{abstract}
Background/Aim: This study aimed to evaluate the outcome of radiation therapy for patients with distant lymph node $(L N)$ metastases, without organ metastases from uterine cervical cancer (UCC). Patients and Methods: Twenty-six patients with UCC with distant $L N$ metastases received radiotherapy and were retrospectively analyzed. The sites of distant LN metastasis were as follows; Supraclavicular in 19, inguinal in nine, axillary in four, and others in three. The mean dose prescribed for these was 50 (range $=40-60$ ) Gy. Results: The 2-year overall, cause-specific, and progressionfree survival, and local control of primary tumor rates were $51.3 \%, 51.3 \%, 46.9 \%$, and $67.9 \%$. In multivariate analysis, performance status $\geq 1$ ( $p=0.007$ ), para-aortic LN metastases $(p=0.001)$, and lack of high-dose-rate intracavitary brachytherapy $(p=0.033)$ were significantly associated with poor overall survival. Performance status $\geq 1 \quad(p=0.004)$, and para-aortic $L N$ metastases $(p=0.014)$ were significantly associated with poor cause-specific survival. Conclusion: This study demonstrated favorable local control in patients with UCC with distant LN metastases.
\end{abstract}

Radiation therapy (RT) has an important role in treating uterine cervical cancer (UCC), especially when at an advanced stage without distant metastasis. However, for cases with

This article is freely accessible online.

Correspondence to: Yuki Mukai (ORCID number: 0000-0002-00001199), MD, Department of Radiation Oncology, Yokohama City University Graduate School of Medicine, Kanazawa-ku, Yokohama, Kanagawa 236-0004, Japan. Tel: +81 457872800, Fax: +81 457860369, e-mail: y_mukai@yokohama-cu.ac.jp

Key Words: Uterine cervical cancer, distant lymph node metastases, supraclavicular lymph node metastases, external beam radiation therapy, three-dimensional radiation therapy. distant metastases, RT of para-aortic lymph nodes (PALNs) has an impact on survival and has been strongly recommended $(1,2)$. In the latest International Federation of Gynecology and Obstetrics (FIGO) Cancer Report 2018, patients with PALN metastases are categorized as having stage IIIC2 disease (previously, they were categorized as stage IVB in the FIGO Cancer Report 2008) (3). The pattern of lymphatic metastatic spread has been demonstrated to be stepwise, from the pelvic LNs to the PALNs, left supraclavicular LNs (SCLNs), and finally the mediastinal LNs. Some tumors spread to the inguinal LNs $(2,4-6)$. These distant LN metastases are still categorized as stage IVB. Management of these stage IVB cases without organ metastases has been controversial. Until recently, the prognosis of patients with stage IVB UCC has been inferior survival, and most of these patients usually receive chemotherapy as initial treatment. However, some reports have found that active treatment/aggressive management of the primary tumor is safe and effective, even in patients with stage IVB UCC with bone or lung metastases (7-9). One report found that patients with stage IVB UCC with only distant LN metastases without organ metastases have better overall (OS) and progression-free (PFS) survival than patients with organ metastases (10). However, it is unclear which distant LN metastases are more dangerous.

We previously investigated the outcomes of RT for UCC with distant metastasis (7-9); however, UCC with distant LN metastases without organ metastases were not considered in detail. In the present study, we therefore retrospectively evaluated response to RT, patterns of treatment failure, and toxicity in patients with UCC with distant LN metastases without organ metastases.

\section{Patients and Methods}

We retrospectively reviewed the medical records of 26 patients who underwent external beam RT, with or without concurrent chemotherapy, for UCC with distant LN metastases without organ 
metastases at our Institution between July 2004 and December 2018. All patients were eligible for this study. Patients who were considered to have stage IIIC2 UCC with only PALN metastases and patients with recurrent cervical cancer were excluded. The clinical stage was based on physical examination, basic laboratory studies, chest X-ray, contrast-enhanced computed tomography (CT) of the neck to the pelvis, and magnetic resonance imaging of the pelvis. One patient was unable to undergo magnetic resonance imaging because of a tattoo. If necessary, bladder and rectum status were determined by cystoscopy and colonoscopy, respectively. All patients were examined before treatment by gynecologists and radiation oncologists, and their disease was classified according to the Union for International Cancer Control staging system (11). Their disease characteristics are summarized in Table I. All patients had both pelvic LN metastases and another site of distant LN metastases: 19 had SCLN metastases, nine had inguinal LN metastases, four had axillary LN metastases, and three had metastases at other sites including the mesentery and mediastinum. Eleven patients had only SCLN metastases. Patients who were considered to have stage IIIC2 UCC with only PALN metastases and patients with recurrent cervical cancer were excluded. This study was approved by our Institutional Review Board (IRB number, B200600006) and informed consent was obtained from all patients before treatment.

Treatment. All 26 patients received three-dimensional RT of the whole pelvic radiation field using 2-4 beams with 15-MV X-rays. Eight patients received whole pelvic RT (WPRT), including bilateral inguinal $\mathrm{LN}$ regions, using two beams. The gross tumor volume was defined as the volume of the primary tumor and involved LNs. The clinical target volume (CTV) was defined as the volume of the primary tumor plus a $5 \mathrm{~mm}$ margin that included the pelvic LNs and the parametrium. The CTV for LNs included the common, external, and internal iliac, and obturator $\mathrm{LN}$ regions and extended to the level of the L4-5 interspace. The CTV for LNs in these eight patients additionally included bilateral inguinal $\mathrm{LN}$ regions. The caudal edge of the radiation field was defined according to the extent of primary disease invasion. The planning target volume was delineated with a $10-15-\mathrm{mm}$ margin around the CTV. Normal structures, including the bladder, rectum, small bowel, and femoral heads, were contoured as organs at risk in the radiation field.

The median prescribed total radiation dose to the pelvis was 50 (range=50-56) Gy, the fraction size was 1.8-2 Gy, and fractions were delivered on 5 consecutive days per week. Fifteen patients $(57.7 \%)$ received high-dose-rate intracavitary brachytherapy (HDRICBT). The remaining patients did not receive HDR-ICBT because their cervical tumors did not shrink sufficiently to warrant intracavitary application or they had comorbidity (dementia, depression), or poor performance status (PS). HDR-ICBT was performed using Ir-192 with microSelectron-HDR (Nucletron, Veenendaal, the Netherlands). The standard regimen of HDR-ICBT in our Institution is 5 Gy per fraction for point A (12).

The median dose of HDR-ICBT was 15 Gy in three fractions (range, 10-25 Gy, in 2-5 fractions). Five patients with pelvic or inguinal $\mathrm{LN}$ involvement, or both, received an additional boost of up to 54-59.4 Gy to those lesions following WPRT. Two patients had an additional boost of up to 56 and 56.4 Gy for their primary tumor. The median duration of all treatments, including external beam radiation therapy and HDR-ICBT, was 46 (range=32-70) days. All patients completed the planned RT for their primary tumors. The total equivalent dose in 2 Gy fractions (EQD2) to the primary tumor
Table I. Patient and treatment related characteristics.

\begin{tabular}{|c|c|c|}
\hline Characteristic & Subgroup & Value \\
\hline Number of patients & Total & 26 \\
\hline Median age, years & Median (range) & $77(41-83)$ \\
\hline \multirow[t]{3}{*}{ ECOG PS, $n$} & 0 & 14 \\
\hline & 1 & 10 \\
\hline & 2 & 2 \\
\hline \multirow{2}{*}{ Histology, $\mathrm{n}$} & Squamous cell carcinoma & 25 \\
\hline & Adenocarcinoma & 1 \\
\hline $\begin{array}{l}\text { Maximum primary tumor } \\
\text { diameter, } \mathrm{mm}\end{array}$ & Median (range) & $60(25-100)$ \\
\hline \multirow[t]{4}{*}{ T-Category, $\mathrm{n}$} & $\mathrm{T} 2 \mathrm{~b}$ & 3 \\
\hline & $\mathrm{T} 3 \mathrm{a}$ & 3 \\
\hline & $\mathrm{T} 3 \mathrm{~b}$ & 16 \\
\hline & $\mathrm{T} 4$ & 4 \\
\hline \multirow[t]{2}{*}{ N-Category, $\mathrm{n}$} & $\mathrm{N} 1$ & 26 \\
\hline & Para-aortic & 21 \\
\hline \multirow{7}{*}{$\begin{array}{l}\text { Site of distant } \mathrm{LN} \\
\text { metastasis, } \mathrm{n}\end{array}$} & With SCLN & 19 \\
\hline & Only SCLN & 11 \\
\hline & SCLN+inguinal & 4 \\
\hline & SCLN+axillary \pm other & 4 \\
\hline & Without SCLN & 7 \\
\hline & Inguinal & 5 \\
\hline & Other & 2 \\
\hline \multirow[t]{2}{*}{ Hydronephrosis, $\mathrm{n}$} & No & 11 \\
\hline & Yes (bilateral) & $13(2)$ \\
\hline \multirow[t]{2}{*}{ Chemotherapy at RT, n } & No & 10 \\
\hline & Concurrent & 16 \\
\hline Chemotherapy before RT, $\mathrm{n}$ & Yes & 2 \\
\hline
\end{tabular}

ECOG PS: Eastern Cooperative Oncology Group performance status; RT: radiation therapy, SCLN: supraclavicular lymph nodes, CRT: chemoradiation therapy. *TNM Classification of Malignant Tumors, eighth edition (11).

$(\alpha / \beta=10)$ was calculated from a linear-quadratic model. The median EQD2 to the primary tumor was 63 (range=48.4-72.4) Gy.

Sixteen out of the 26 patients had chemotherapy concomitant with RT, consisting of weekly cisplatin $\left(\mathrm{n}=15 ; 40 \mathrm{mg} / \mathrm{m}^{2}\right.$ body surface area) or paclitaxel $\left(135 \mathrm{mg} / \mathrm{m}^{2}\right.$ ) and carboplatin (area under the curve 5, Calvert's formula) in 1 . The reasons for the remaining 10 patients not receiving chemotherapy were as follows: Renal dysfunction in four; poor general condition (poor PS), old age, or comorbidity in 5; and patient refusal in one.

At initial diagnosis of UCC, 21 patients had PALN metastases, and 15 of them received RT to the PALN region immediately after initial WPRT within 2 months. The median RT dose was 52.2 (range $=40-60$ ) Gy, delivered using two or four 15-MV beams. In the remaining six patients, the involved PALNs had become unclear after concurrent chemoradiation therapy in five, and induction chemotherapy in one for the primary tumor, and they did not receive PALN RT. One patient developed PALN metastases 6 months after the initial treatment, and she received RT of 54 Gy in 27 fractions.

At initial diagnosis, 19 patients had left SCLN metastases (Table I), and 14 of them received RT for the metastases at a median dose of 50 (range=40-60) Gy. RT was delivered using the parallel-opposed (antero-posterior) field at $6 \mathrm{MV}$. Thirteen patients received RT within 2 months after initial WPRT, and only 
os

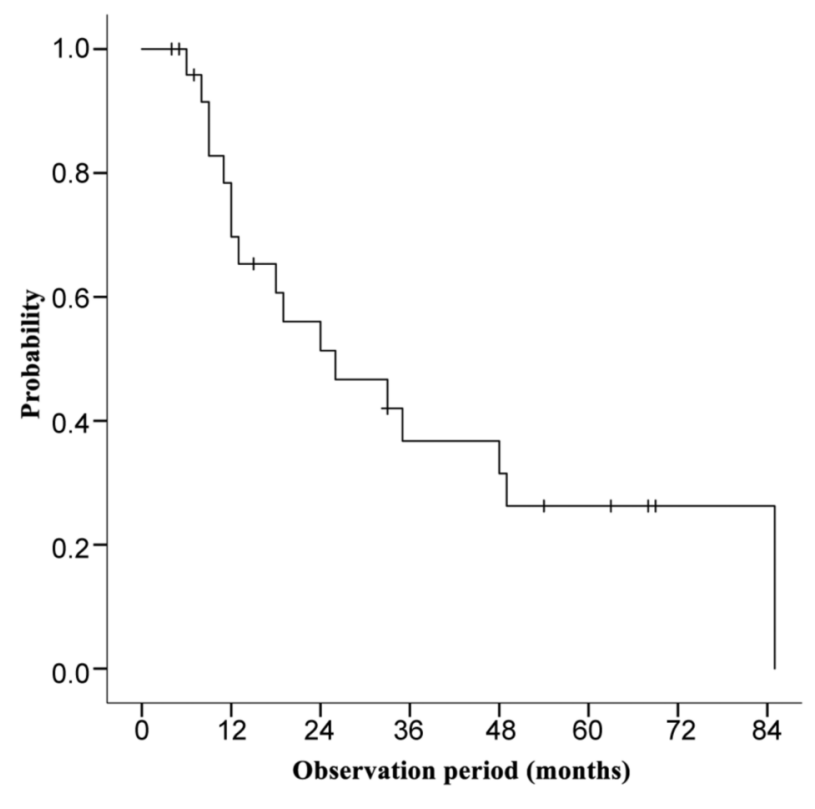

PFS

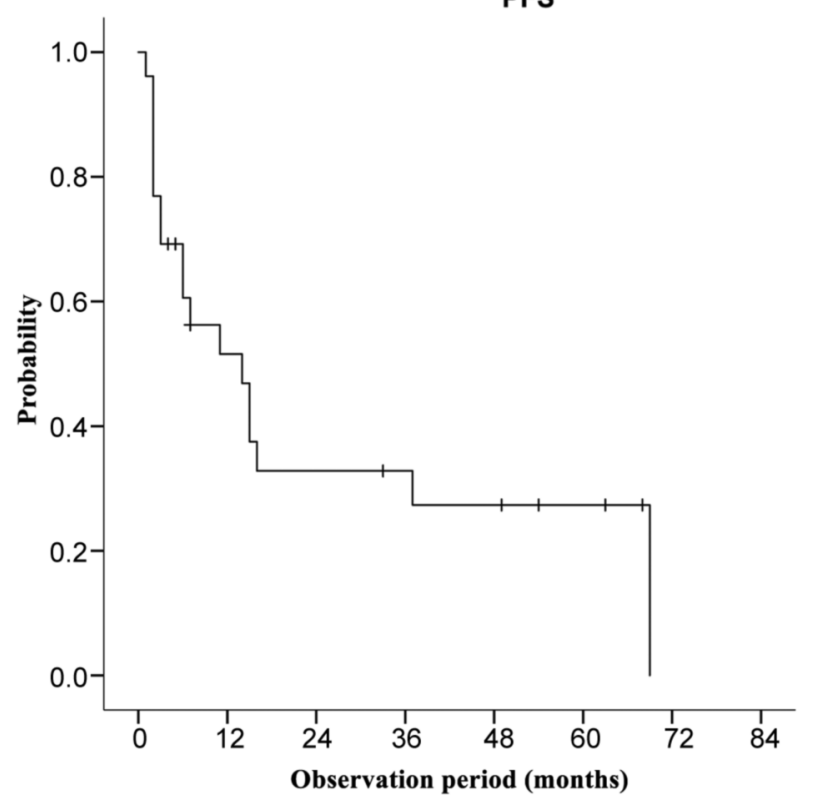

cSS

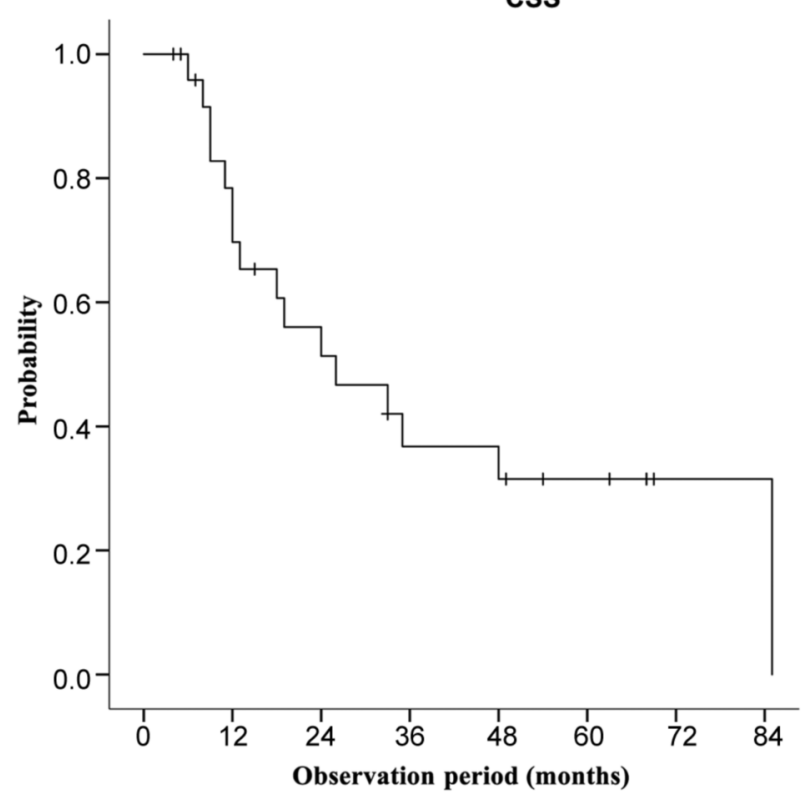

LC

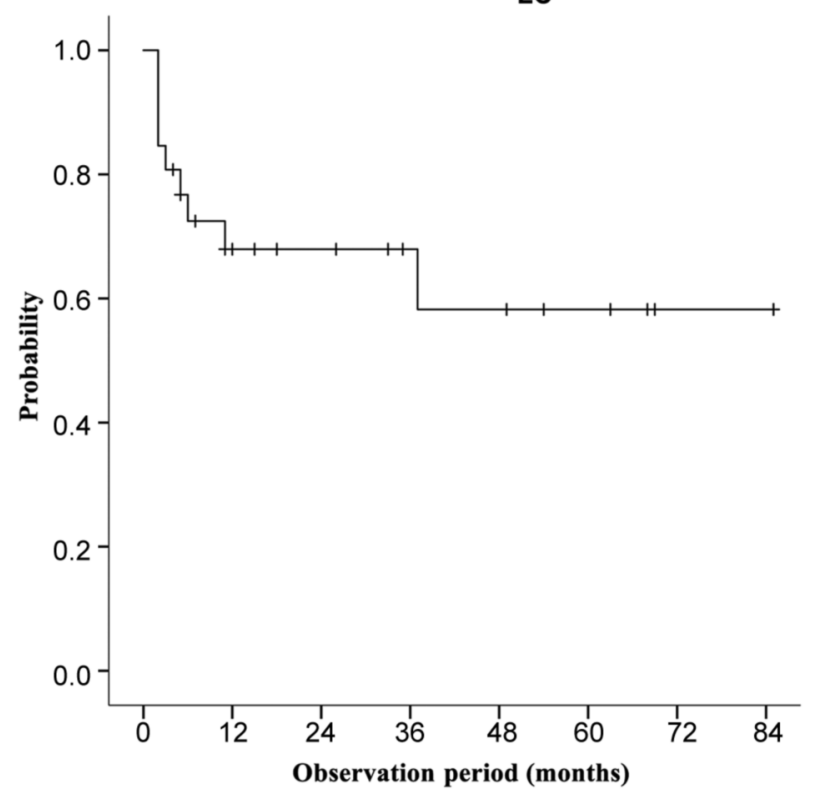

Figure 1. Kaplan-Meier curves for overall (OS) (A), cause-specific (CSS) (B) and progression-free (PFS) (C) survival, and local control (LC) of primary tumor $(D)$.

one patient received RT at the same time as initial WPRT. The remaining five patients did not receive RT for SCLN metastases. In three who received initial concurrent chemoradiotherapy for the primary tumor and one who received induction chemotherapy, their SCLNs became undetectable after initial treatment; therefore, they did not undergo RT of the SCLN region. The other patient developed disseminated intravascular coagulation soon after initial treatment and missed the chance of RT for SCLN metastases. Nine patients had inguinal LN metastases, and eight received RT for the metastases at a median dose of 54.9 (range=50.4-59.4) Gy, at the same time as initial WPRT. The inguinal LNs were usually irradiated by enlarging the field of pelvic RT. One patient did not receive RT for inguinal LNs because she had SCLN metastasis and curative treatment was considered difficult. One out of four patients who had axillary LN metastases received RT by enlarging the field of SCLN, and in the remaining three patients, axillary $\mathrm{LN}$ metastases became undetectable after initial treatment. 
Evaluation criteria and statistical analysis. Responses were evaluated by clinical examination and CT from the neck to the pelvis within 4-6 weeks following the completion of treatment. Tumor responses were assessed using the Response Evaluation Criteria in Solid Tumors (RECIST ver. 1.1) (13). Treatmentassociated toxicity was evaluated using the Common Terminology Criteria for Adverse Events v4.0 (14). Acute toxicity was defined as treatment-related adverse events that occurred within 3 months after initiation of treatment, and late toxicity as adverse events occurring after 3 months. OS, cause-specific survival (CSS), local control for primary tumor (LC), and PFS rates from initiation of treatment were calculated with Kaplan-Meier curves. Differences between curves were tested by the log-rank test. Analyses of prognostic factors used univariate and multivariate Cox proportional-hazards regression models, with SPSS for Windows version 23.0 (IBM, Armonk, NY, USA). A value of $p<0.05$ was considered significant.

\section{Results}

Survival and tumor control. The median follow-up time was 18.5 (range=4-85) months. Two-year OS, CSS, PFS and LC rates were $51.3 \%, 51.3 \%, 46.9 \%$, and $67.9 \%$, respectively (Figure 1). In 17 out of 26 patients, the primary tumor was controlled or showed a complete response at the last evaluation. Among the remaining nine patients, three developed progression of their primary tumors and one had recurrence in the pelvic wall, which was confirmed by CT. The three patients with malignant cells only were identified by Papanicolaou smear but these were not detected by imaging; one patient had residual tumor, which remained as stable disease, and another patient developed recurrence in the vagina. In the three patients with progression of the primary tumor, their EQD2 was under the median value, and two did not receive HDR-ICBT. One patient developed both vesicovaginal and rectovaginal fistulas because of invasion of the recurrent tumor. At the last follow-up, 16 patients had died of UCC, nine were alive and disease-free, and one had died in a traffic accident but was considered disease-free. The median survival of these 10 disease-free patients was 41 (range=4-69) months. Eleven patients died of distant metastases (lung, $\mathrm{n}=5$; liver and/or peritoneal seeding, $\mathrm{n}=3$; or mediastinal LNs, $n=3$ ), and five died of local or locoregional metastases (residual tumor, $n=2$; pelvic tumor, $\mathrm{n}=2$; or vaginal recurrence, $\mathrm{n}=1$ ).

Control of irradiated distant LN metastases. Eight patients received RT for inguinal LN metastases, which were controlled in seven patients. Fourteen out of 19 patients with SCLN metastases received RT, and 12 achieved complete response and two partial response. Among five patients who did not receive sequential RT after WPRT, one changed hospital soon after the initial treatment and we were unable to obtain further information. All four remaining patients developed SCLN relapse and developed axillary or

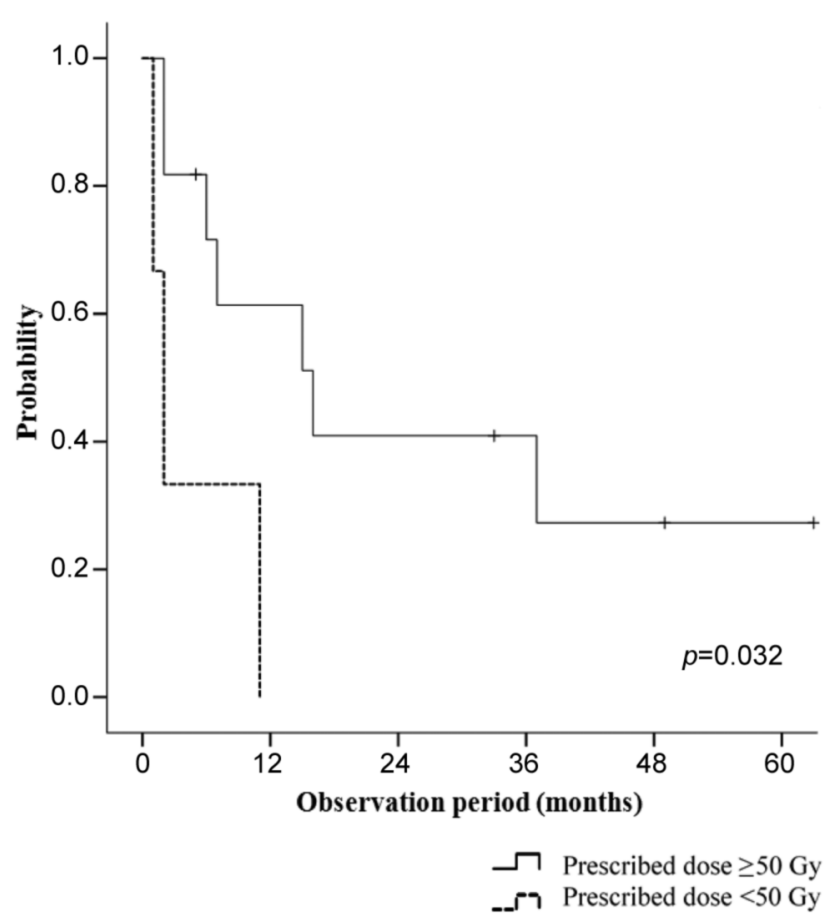

Figure 2. Progression-free survival according to the prescribed dose for supraclavicular lymph nodes.

mediastinal LN metastases. Three of them received RT after tumor recurrence. However, two patients developed metastases of other organs, and SCLN metastases were considered to indicate progressive disease. Control of SCLN metastases was achieved in only one patient but she developed other organ metastases approximately 4 years after SCLN irradiation. In the 14 patients who received RT for SCLN metastases, those prescribed radiation doses $<50$ Gy demonstrated significantly inferior PFS ( $p=0.032$, Figure 2$)$. The prescribed dose had no significant effect on OS $(p=0.105), \operatorname{CSS}(p=0.105)$, and control of SCLN metastases $(p=0.082)$.

Analysis of prognostic factors. In univariate analysis, EQD2 greater than the median value was significantly associated with better OS $(p=0.037)$ and LC $(p=0.034)$, and SCLN metastases was significantly associated with poorer OS $(p=0.031), \operatorname{CSS}(p=0.011)$, and PFS ( $p=0.007)$ (Tables II and III). In multivariate analysis, Eastern Cooperative Oncology Group PS $\geq 1$ [hazard ratio $(\mathrm{HR})=17.3,95 \%$ confidence interval $(\mathrm{CI})=1.6-183.4 ; \quad p=0.007] ; \quad$ PALN metastases $(\mathrm{HR}=104.9,95 \% \mathrm{CI}=2.9-3860.4 ; p=0.001)$, and no HDRICBT $(\mathrm{HR}=2.5,95 \% \mathrm{CI}=0.9-6.9 ; p=0.033)$ were significantly associated with poor OS. Moreover, $\mathrm{PS} \geq 1$ ( $\mathrm{HR}=22.6,95 \%$ $\mathrm{CI}=1.9-259.2 ; p=0.004)$ and PALN metastases $(\mathrm{HR}=63.7$, $95 \% \mathrm{CI}=1.5-2649.5 ; p=0.014)$ were significantly associated with poor CSS. SCLN metastases were significantly 
Table II. Univariate analysis of prognostic factors.

\begin{tabular}{|c|c|c|c|c|c|c|c|c|c|}
\hline \multirow[t]{2}{*}{ Variable } & \multirow[t]{2}{*}{ Subgroup } & \multicolumn{2}{|l|}{ OS } & \multicolumn{2}{|l|}{ CSS } & \multicolumn{2}{|l|}{ PFS } & \multicolumn{2}{|l|}{ LC } \\
\hline & & HR $(95 \%$ CI) & $p$-Value & HR $(95 \%$ CI $)$ & $p$-Value & HR $(95 \%$ CI $)$ & $p$-Value & HR $(95 \%$ CI $)$ & $p$-Value \\
\hline \multirow[t]{2}{*}{ Age } & $<77$ Years & 1 & & 1 & & 1 & & 1 & \\
\hline & $\geq 77$ Years & $1.00(0.284-3.52)$ & $>0.999$ & $0.659(0.148-2.93)$ & 0.565 & $0.480(0.11-2.11)$ & 0.287 & $1.107(0.229-5.36$ & 0.900 \\
\hline \multirow[t]{2}{*}{ ECOG PS } & 0 & 1 & & 1 & & 1 & & 1 & \\
\hline & $>1$ & $2.217(0.798-6.15)$ & 0.136 & $2.42(0.848-6.91)$ & 0.106 & $1.66(0.627-4.43)$ & 0.311 & $2.124(0.549-8.21)$ & 0.273 \\
\hline \multirow[t]{2}{*}{ T-Category } & $\leq \mathrm{T} 3 \mathrm{a}$ & 1 & & 1 & & 1 & & 1 & \\
\hline & $>\mathrm{T} 3 \mathrm{a}$ & $4.81(0.633-36.49)$ & 0.056 & $4.32(0.567-32.95)$ & 0.080 & $1.65(0.472-5.76)$ & 0.408 & $2.45(0.306-19.63)$ & 0.344 \\
\hline \multirow{2}{*}{$\begin{array}{l}\text { Primary tumor } \\
\text { diameter }\end{array}$} & $\leq 60 \mathrm{~mm}$ & 1 & & 1 & & 1 & & 1 & \\
\hline & $>60 \mathrm{~mm}$ & $1.786(0.665-4.80)$ & 0.253 & $1.62(0.584-4.50)$ & 0.936 & $1.48(0.557-3.91)$ & 0.437 & $2.98(0.734-12.10)$ & 0.113 \\
\hline \multirow[t]{2}{*}{ PAN metastasis } & No & 1 & & 1 & & 1 & & 1 & \\
\hline & Yes & $4.24(0.558-32.23)$ & 0.084 & $3.76(0.494-28.7)$ & 0.121 & $2.47(0.561-10.82)$ & 0.182 & $2.23(0.278-17.87)$ & 0.404 \\
\hline \multirow{2}{*}{$\begin{array}{l}\text { SCLN } \\
\text { metastasis }\end{array}$} & No & 1 & & 1 & & 1 & & 1 & \\
\hline & Yes & $4.18(0.920-18.97)$ & 0.0309 & $7.48(0.963-57.29)$ & 0.0110 & $5.425(1.22-24.10)$ & 0.00743 & $4.50(0.552-36.65)$ & 0.935 \\
\hline \multirow{2}{*}{$\begin{array}{l}\text { Primary tumor } \\
\text { EQD2 }\end{array}$} & $\leq$ Median & 1 & & 1 & & 1 & & 1 & \\
\hline & > Median & $0.343(0.123-0.957)$ & 0.0369 & $0.386(0.135-1.100)$ & 0.0714 & $0.541(0.203-1.44)$ & 0.213 & $4.71(0.965-22.98)$ & 0.0336 \\
\hline \multirow[t]{2}{*}{ HDR-ICBT } & No & 1 & & 1 & & 1 & & 1 & \\
\hline & Yes & $0.395(0.146-1.07)$ & 0.0727 & $0.447(0.159-1.26)$ & 0.134 & $0.661(0.253-1.73)$ & 0.402 & $0.297(0.073-1.20)$ & 0.0764 \\
\hline \multirow[t]{2}{*}{ Chemotherapy } & No & 1 & & 1 & & 1 & & 1 & \\
\hline & Yes & $0.907(0.327-2.52)$ & 0.853 & $1.05(0.355-3.075)$ & 0.936 & $1.20(0.442-3.250)$ & 0.719 & $0.457(0.122-1.71)$ & 0.243 \\
\hline \multirow{2}{*}{$\begin{array}{l}\text { Irradiated } \\
\text { distant LN } \\
\text { metastasis }\end{array}$} & No & 1 & & 1 & & 1 & & 1 & \\
\hline & Yes & $1.11(0.323-4.015)$ & 0.837 & $1.07(0.300-3.80)$ & 0.918 & $0.738(0.240-2.27)$ & 0.607 & $0.755(0.157-3.64)$ & 0.733 \\
\hline
\end{tabular}

OS: Overall survival; CSS: cause-specific survival; PFS: progression-free survival; HR: hazard ratio; CI: confidence interval; ECOG PS: Eastern Cooperative Oncology Group performance status; LN: lymph node; PAN: para-aortic lymph nodes; SCLN: supraclavicular lymph nodes; EQD2: 2 Gy per fraction equivalent dose; HDR-ICBT: high-dose-rate intracavitary brachytherapy. Significant $p$-Values are indicated in bold.

associated with poor survival $(\mathrm{OS}$ : $\mathrm{HR}=16.1,95 \% \mathrm{CI}=1.4-$ 179.9, $p=0.018 ; \mathrm{CSS}: \mathrm{HR}=26.4,95 \% \mathrm{CI}=1.5-453.9, p=0.011$; and PFS: $\mathrm{HR}=7.9,95 \% \mathrm{CI}=0.73-87.4, p=0.049)$. No factor had a significant effect on LC of primary tumor in multivariate analysis (data not shown).

Toxicity. For acute toxicity, only hematological toxicity was grade or more (Table IV). Only grade 1 acute gastrointestinal toxicity was observed. Fourteen patients who received sequential RT for SCLN metastases, and three who received RT for SCLN metastases after recurrence developed grade 1 or 2 radiation dermatitis around the SCLN region. For inguinal LNs, half of the patients who received RT developed radiation dermatitis. All acute hematological and gastrointestinal toxicity was tolerable or manageable. For late toxicity, only one patient developed grade 1 rectal hemorrhage and this symptom was short-lived.

\section{Discussion}

Among distant metastases from UCC, PALN metastases respond well to (concurrent chemo)RT and show promising favorable prognosis $(1,2)$. Therefore, in the latest FIGO Cancer Report 2018 (3), patients with UCC with PALN metastases became categorized as stage IIIC2. Patients with other distant metastases, whether organ or lymphatic, have been categorized as stage IVB. In previous studies, the 5-year OS was reported as $9-16.5 \%$ in patients diagnosed with stage IVB UCC (15-18). In lung metastases from UCC, the 5-year survival was reported as $10-60 \%$ (19-24). The 2-year PFS rate was $34 \%$ in patients with bone metastases from UCC (7). Lung metastases are the most frequently detected and there are few reports about brain or liver metastases (25).

Jung Ho Im et al. compared the treatment outcome between patients with only distant LN metastases or metastases of visceral organs and demonstrated that those with only distant LN metastases experienced significantly better PFS and OS (10). The outcomes of RT for patients with SCLN metastases were reported as 2-year OS of 17-36\%, 3year OS of 49-66.7\%, 5-year OS of 46.3-55.6\%, and 3-year PFS of $35.3-66.7 \%(6,25,26)$. In our study, the 2-year OS of $51.3 \%$ and PFS of $46.9 \%$ were comparable to those of previous reports about distant LN metastases. Our study showed a favorable prognosis for patients with only distant LN metastases compared with visceral organ metastasis. The most common cause of death was metastases in the lungs in 11 and mediastinum in three, and how to control these organ metastases may be key to improving survival. 
Table III. Multivariate analysis of prognostic factors.

\begin{tabular}{|c|c|c|c|c|c|c|c|}
\hline \multirow[t]{2}{*}{ Variable } & \multirow[t]{2}{*}{ Subgroup } & \multicolumn{2}{|l|}{ OS } & \multicolumn{2}{|l|}{ CSS } & \multicolumn{2}{|l|}{ PFS } \\
\hline & & HR $(95 \%$ CI $)$ & $p$-Value & HR $(95 \%$ CI $)$ & $p$-Value & $\mathrm{HR}(95 \% \mathrm{CI})$ & $p$-Value \\
\hline \multirow[t]{2}{*}{ Age, years } & $<77$ Years & 1 & & 1 & & 1 & \\
\hline & $\geq 77$ Years & $2.94(0.425-20.32)$ & 0.272 & $5.75(0.017-58.7)$ & 0.119 & $4.21(0.626-28.3)$ & 0.096 \\
\hline \multirow[t]{2}{*}{ ECOG PS } & 0 & 1 & & 1 & & 1 & \\
\hline & $>1$ & $17.33(1.64-183.4)$ & 0.0068 & $22.57(1.97-259.2)$ & 0.0039 & $2.92(0.501-16.98)$ & 0.225 \\
\hline \multirow[t]{2}{*}{ T-Category } & $\leq \mathrm{T} 3 \mathrm{a}$ & 1 & & 1 & & 1 & \\
\hline & $>\mathrm{T} 3 \mathrm{a}$ & $7.02(0.598-82.35)$ & 0.066 & $5.99(0.521-68.78)$ & 0.093 & $1.025(0.251-4.18)$ & 0.972 \\
\hline \multirow[t]{2}{*}{ PAN metastasis } & No & 1 & & 1 & & 1 & \\
\hline & Yes & $104.9(2.85-3860.4)$ & 0.0013 & $63.66(1.53-2649.5)$ & 0.0136 & $1.53(0.135-17.22)$ & 0.732 \\
\hline \multirow[t]{2}{*}{ SCLN metastasis } & No & 1 & & 1 & & 1 & \\
\hline & Yes & $16.06(1.43-179.9)$ & 0.0175 & $26.45(1.54-453.9)$ & 0.0106 & $7.97(0.727-87.37)$ & 0.0490 \\
\hline \multirow[t]{2}{*}{ Primary tumor EQD2 } & $\leq$ Median & 1 & & 1 & & 1 & \\
\hline & $>$ Median & $0.340(0.11-5.19)$ & 0.789 & $0.786(0.122-5.07)$ & 0.804 & $0.532(0.100-2.82)$ & 0.479 \\
\hline \multirow[t]{2}{*}{ HDR-ICBT } & No & 1 & & 1 & & 1 & \\
\hline & Yes & $0.132(0.017-1.00)$ & 0.0334 & $0.193(0.022-1.69)$ & 0.129 & $1.07(0.115-10.00)$ & 0.952 \\
\hline \multirow{2}{*}{ Chemotherapy } & No & 1 & & 1 & & 1 & \\
\hline & Yes & $0.292(0.037-2.30)$ & 0.237 & $0.218(0.025-1.93)$ & 0.161 & $0.727(0.139-3.80)$ & 0.705 \\
\hline
\end{tabular}

OS: Overall survival; CSS: cause-specific survival; PFS: progression-free survival; HR: hazard ratio; CI: confidence interval; ECOG PS: Eastern Cooperative Oncology Group performance status; PAN: para-aortic lymph nodes; SCLN: supraclavicular lymph nodes; EQD2: 2 Gy per fraction equivalent dose; HDR-ICBT: high-dose-rate intracavitary brachytherapy. Significant $p$-Values are indicated in bold.

In the current study, RT controlled inguinal LN metastases (complete or partial response) in seven out of eight patients and SCLN metastases in 14 out of 19 patients. Five patients did not receive sequential RT. One patient changed hospital, and the other four did not receive sequential RT for SCLN metastases because their SCLNs became undetectable after RT for their primary tumor. All of them developed SCLN recurrence and metastases of other LNs and organs. In cases where the SCLNs seemed shrunken after initial treatment, there was a possibility that microscopic malignant cells remained in the $\mathrm{LN}$ regions, and we recommend considering prophylactic irradiation.

Lee et al. reported that a prescription dose <66 Gy achieved good control of SCLN metastases; however, their study included only seven patients (25). They concluded that the RT dose for local control of SCLN metastases has not yet been established. In our study, no significant relationship was observed between the control of SCLN metastases and the prescribed radiation dose. However, our study demonstrated that a prescribed dose of $<50$ Gy for SCLN metastases was significantly correlated with inferior PFS. However, it is hard to state for sure what the optimum prescription dose for SCLN metastases is at this point because of the small number of patients included in our study. Currently, we only suggest that it may be better to consider a prescription dose of $\geq 50$ Gy for SCLN metastases, in accordance with the report by Lee et al. Large studies are required to determine the optimum prescription dose for SCLN metastases.
Table IV. Acute toxicity experienced by patients in this study.

\begin{tabular}{|c|c|c|c|c|c|c|}
\hline \multirow[b]{2}{*}{ Acute toxicity } & & \multicolumn{5}{|c|}{ Grade, $\mathrm{n}$} \\
\hline & & 1 & 2 & 3 & 4 & 5 \\
\hline \multirow[t]{4}{*}{ Hematology } & Anemia & 3 & 15 & 4 & 0 & 0 \\
\hline & Leukocytes/leukopenia & 1 & 9 & 9 & 0 & 0 \\
\hline & Neutropenia & 3 & 5 & 3 & 1 & 0 \\
\hline & Thrombocytopenia & 0 & 0 & 2 & 0 & 0 \\
\hline \multirow[t]{3}{*}{ Dermatitis } & Inguinal & 3 & 1 & 0 & 0 & 0 \\
\hline & Vulvar & 1 & 1 & 0 & 0 & 0 \\
\hline & SCLN & 14 & 3 & 0 & 0 & 0 \\
\hline \multirow[t]{2}{*}{ Mucositis } & Vulval & 2 & 0 & 0 & 0 & 0 \\
\hline & Pharyngeal/laryngeal & 7 & 0 & 0 & 0 & 0 \\
\hline \multirow[t]{4}{*}{ Gastrointestinal } & Anorexia & 1 & 0 & 0 & 0 & 0 \\
\hline & Diarrhea & 9 & 0 & 0 & 0 & 0 \\
\hline & Nausea & 0 & 0 & 0 & 0 & 0 \\
\hline & Rectal hemorrhage & 0 & 0 & 0 & 0 & 0 \\
\hline \multirow{2}{*}{ Genitourinary } & Urinary frequency & 0 & 0 & 0 & 0 & 0 \\
\hline & Cystitis, non-infective & 0 & 0 & 0 & 0 & 0 \\
\hline
\end{tabular}

SCLN: Supraclavicular lymph node.

Kim et al. reported that chemoradiation significantly improved OS and PFS in patients with stage IVB UCC, and that squamous cell histology was a significant prognostic factor for favorable PFS (26). In our study, no significant difference was found in chemoradiation. PALN metastases were significantly associated with OS and CSS in 
multivariate analysis $(1,2)$. Our study found that SCLN metastases were significantly associated with poor OS, CSS, and PFS. However, irradiation for distant LN metastases had no significant effect on survival.

In our study, the most common toxicity was hematological, which was comparable to previous reports $(25,26)$. Dermatitis was almost inevitable for LN irradiation; however, it was all low grade (1 or 2) and manageable.

The limitations of our study included its retrospective nature, short observation period, and small number of patients because of disease specificity. We cannot deny the possibility of some bias. Some of the patients were treated for LN metastases with intent of palliative or symptomatic relief but not active curative intent in the first stage of this study in the prescribed doses for distant metastases LNs and HDR-ICBT.

\section{Conclusion}

This study demonstrated favorable local control in patients with stage IVB UCC with distant LN metastases, and that SCLN metastasis was a prognostic factor for OS, CSS, and PFS. Further studies are needed to confirm the treatment, and to determine long-term efficacy and late toxicity.

\section{Conflicts of Interest}

None of the Authors have any conflicts of interest associated with this study.

\section{Authors' Contributions}

Conceptualization, analysis and writing-original draft preparation: Y.M, and M.H. Writing - review and editing: All Authors.

\section{References}

1 Niibe Y, Kenjo M, Kazumoto T, Michimoto K, Takayama M, Yamauchi C, Kataoka M, Suzuki K, Ii N, Uno T, Takanaka T, Higuchi K, Yamazaki H, Tokumaru S, Oguchi M and Hayakawa $\mathrm{K}$ : Multi-institutional study of radiation therapy for isolated para-aortic lymph node recurrence in uterine cervical carcinoma: 84 Subjects of a population of more than 5,000. Int J Radiat Oncol Biol Phys 66: 1366-1369, 2006. PMID: 17126206. DOI: 10.1016/j.ijrobp.2006.07.1384

2 Kim YS, Kim JH, Ahn SD, Lee SW, Shin SS, Nam JH, Kim YT, Kim YM, Kim JH and Choi EK: High-dose extended-field irradiation and high-dose-rate brachytherapy with concurrent chemotherapy for cervical cancer with positive para-aortic lymph nodes. Int J Radiat Oncol Biol Phys 74: 1522-1528, 2009. PMID: 19231106. DOI: 10.1016/j.ijrobp.2008.10.024

3 Bhatla N, Aoki D, Sharma DN and Sankaranarayanan R: FIGO Cancer Report 2018: Cancer of the cervix uteri. Int J Gynecol Obstet 143(S2): 22-36, 2018. PMID: 30306584. DOI: 10.1002/ ijgo. 12611

4 Sakuragi N, Satoh C, Takeda N, Hareyama H, Takeda M, Yamamoto R, Fujimoto T, Oikawa M, Fujino T and Fujimoto S:
Incidence and distribution pattern of pelvic and paraaortic lymph node metastasis in patients with stages IB, IIA, and IIB cervical carcinoma treated with radical hysterectomy. Cancer 85 : 15471554, 1999. PMID: 10193945. DOI: 10.1002/(sici)10970142(19990401)85:7<1547::aid-cncr16>3.0.co;2-2

5 Stehman FB, Bundy BN, Hanjani P, Fowler WC, Abdulhay G and Whitney CW: Biopsy of the scalene fat pad in carcinoma of the cervix uteri metastatic to the periaortic lymph nodes. Surg Gynecol Obstet 165: 503-506, 1987. PMID: 3686315.

6 Ning MS, Ahobila V, Jhingran A, Stecklein SR, Frumovitz M, Schmeler KM, Eifel PJ and Klopp AH: Outcomes and patterns of relapse after definitive radiation therapy for oligometastatic cervical cancer. Gynecol Oncol 148: 132-138, 2018. PMID: 29089122. DOI: 10.1016/j.ygyno.2017.10.017

7 Hata M, Koike I, Miyagi E, Asai-Sato M, Kaizu H, Mukai Y, Takano S, Ito E, Sugiura M and Inoue T: Radiation therapy for patients with bone metastasis from uterine cervical cancer: Its role and optimal radiation regimen for palliative care. Anticancer Res 38: 1033-1040, 2018. PMID: 29374737. DOI: 10.21873/ anticanres.12319.

8 Mukai Y, Koike I, Matsunaga T, Ruiz-Yokota N, Takano S, Sugiura M, Sato M, Miyagi E and Hata M: Radiation therapy for uterine cervical cancer with lung metastases including oligometastases. In Vivo 33: 1677-1684, 2019. PMID: 31471423. DOI: 10.21873 /invivo. 11655

9 Hata M, Omura M, Miyagi E, Koike I, Numazaki R, Asai-Sato M, Tayama Y, Ogino I, Hirahara F and Inoue T: The role of radiation therapy for uterine cervical cancer with distant metastasis. Oncology 83: 67-74, 2012. PMID: 22760158. DOI: 10.1159/000337985

$10 \mathrm{Im}$ JH, Yoon HI, Kim S, Nam EJ, Kim SW, Yim GW, Keum KC, Kim YT, Kim GE and Kim YB: Tailored radiotherapeutic strategies for disseminated uterine cervical cancer patients. Radiat Oncol 10: 77, 2015. PMID: 25884833. DOI: 10.1186/ s13014-015-0373-0

11 Brierley JD, Gospodarowicz MK and Wittekind C (eds.): TNM Classification of Malignant Tumours, Eighth Edition 2017. John Wiley \& Sons, Ltd.

12 Tod MC and Meredith WJ: A dosage system for use in the treatment of cancer of the uterine cervix. Br J Radiol 11: 809824, 1938. DOI: 10.1259/0007-1285-11-132-809

13 Response Evaluation Criteria in Solid Tumor (RECIST) 1.1. Available at: https://recist.eortc.org/recist-1-1-2/ [Last accessed on October 12th 2020 ]

14 The Common Terminology Criteria for Adverse Events (CTCAE) v4.0. Available at: https://ctep.cancer.gov/protocol Development/electronic_applications/ctc.htm\#ctc_40 [Last accessed on October 12th, 2020$]$

15 Quinn MA, Benedet JL, Odicino F, Maisonneuve P, Bellerv U, Creasman WT, Heintz APM, Ngan HYS and Pecorelli S: Carcinoma of the cervix uteri. FIGO 26 th Annual Report on the results of treatment in gynecological cancer. Int J Gynaecol Obstet 95: S43-S103, 2006. PMID: 17161167. DOI: 10.1016/S00207292(06)60030-1

16 Ishikawa M, Nakayama K, Rahman MT, Rahman M, Katagiri A, Katagiri H, Ishibashi T, Iida K and Miyazaki K: A case of stage $\mathrm{IVb}$ cervical carcinoma in which survival was prolonged by two different chemotherapies and CCRT. Gan To Kagaku Ryoho 39: 451-455, 2012. PMID: 22421778.

17 Lim MC, Lee HS, Seo SS, Kim MS, Kim JY, Zo J and Park SY: Pathologic diagnosis and resection of suspicious thoracic 
metastases in patients with cervical cancer through thoracotomy or video-assisted thoracic surgery. Gynecol Oncol 116: 478-482, 2010. PMID: 19889448. DOI: 10.1016/j.ygyno. 2009.10.039

18 Seki M, Nakagawa K, Matsubara T, Kinoshita I, Weng SY and Tsuchiya E: Surgical treatment of pulmonary metastases from uterine cervical cancer. Operation method by lung tumor size. J Thorac Cardiovasc Surg 104: 876-881, 1992. PMID: 1405684.

19 Casaurrán G, Adiego SC, Pascual RP, Mata NM, Barriuso MÁL and Aragoneses FG: Surgery of female genital tract tumour lung metastases. Arch Bronconeumol 47: 134-137, 2011. PMID: 21392876. DOI: 10.1016/j.arbres.2010.10.013

20 Clavero JM, Deschamps C, Cassivi SD, Allen MS, Nichols $3^{\text {rd }}$ FC, Barrette BA, Larson DR and Pairolero PC: Gynecologic cancers: factors affecting survival after pulmonary metastasectomy. Ann Thorac Surg 81: 2004-2007, 2006. PMID: 16731120. DOI: 10.1016/j.athoracsur.2006.01.068

21 Mountain CF, McMurtrey MJ and Hermes KE: Surgery for pulmonary metastasis: a 20-year experience. Ann Thorac Surg 38: 323-330, 1984. PMID: 6486949. DOI: 10.1016/s00034975(10)62280-1

22 Hwang JH, Yoo HJ, Lim MC, Seo SS, Kang S, Kim JY and Park SY: Brain metastasis in patients with uterine cervical cancer. J Obstet Gynaecol Res 39: 287-291, 2013. PMID: 22690955. DOI: $10.1111 / \mathrm{j} .1447-0756.2012 .01927 . \mathrm{x}$
$23 \mathrm{Li} \mathrm{H}, \mathrm{Wu} \mathrm{X}$ and Cheng X: Advances in diagnosis and treatment of metastatic cervical cancer. J Gynecol Oncol 27: e43, 2016. PMID: 27171673. DOI: 10.3802/jgo.2016.27.e43

$24 \mathrm{Kim}$ JY, Kim JH, Yoon MS and Kim J: Curative chemoradiotherapy in patients with stage IVB cervical cancer presenting with paraortic and left supraclavicular lymph node metastases. Int J Radiat Oncol Biol Phys 84: 741-747, 2012. PMID: 22898382. DOI: 10.1016/j.ijrobp.2012.01.070

25 Lee SH, Lee SH, Lee KC, Lee KB, Shin JW, Park CY, Sym SJ and Lee JH: Radiation therapy with chemotherapy for patients with cervical cancer and supraclavicular lymph node involvement. J Gynecol Oncol 23: 159-67, 2012. PMID: 22808358. DOI: $10.3802 /$ jgo.2012.23.3.159

26 Kim HS, Kim T, Lee ES, Kim HJ, Chung HH, Kim JW, Song YS and Park NH: Impact of chemoradiation on prognosis in stage 4B cervical cancer with distant lymphatic metastasis. Cancer Res Treat 45: 193-201, 2013. PMID: 24155678. DOI: 10.4143/crt.2013.45.3.193

Received December 9, 2020

Revised December 25, 2020

Accepted January 5, 2021 